\title{
Local Fractional Z-Transforms with Applications to Signals on Cantor Sets
}

\author{
Kai Liu, ${ }^{1}$ Ren-Jie Hu, ${ }^{1}$ Carlo Cattani, ${ }^{2}$ Gong-Nan Xie, ${ }^{3}$ Xiao-Jun Yang, ${ }^{4}$ and Yang Zhao ${ }^{5}$ \\ ${ }^{1}$ School of Electrical Engineering, Southeast University, Nanjing 210096, China \\ ${ }^{2}$ Department of Mathematics, University of Salerno, Via Ponte don Melillo, Fisciano, 84084 Salerno, Italy \\ ${ }^{3}$ School of Mechanical Engineering, Northwestern Polytechnical University, Xi'an, Shaanxi 710048, China \\ ${ }^{4}$ Department of Mathematics and Mechanics, China University of Mining and Technology, Xuzhou, Jiangsu 221008, China \\ ${ }^{5}$ Department of Electronic and Information Technology, Jiangmen Polytechnic, Jiangmen 529090, China
}

Correspondence should be addressed to Yang Zhao; zhaoyang19781023@gmail.com

Received 28 January 2014; Accepted 3 February 2014; Published 10 March 2014

Academic Editor: Ming Li

Copyright (c) 2014 Kai Liu et al. This is an open access article distributed under the Creative Commons Attribution License, which permits unrestricted use, distribution, and reproduction in any medium, provided the original work is properly cited.

The $Z$-transform has played an important role in signal processing. In this paper the $Z$-transform has been generalized by the coupling of both the $Z$-transform and the local fractional complex calculus. In the literature the local fractional $Z$-transform is applied to analyze signals, in the following it will be used to analyze signals on Cantor sets. Some examples are also given to show the efficiency and accuracy for handling the signals on Cantor sets.

\section{Introduction}

Integral transforms [1, 2], such as Fourier, Laplace, Mellin, Hilbert, and Hankel transforms, play important roles in solving the mathematical problems arising in applied mathematics, mathematical physics, and engineering science. In recent years, fractional calculus [3-11] was developed and used to model also some anomalous behaviors of diffusion [12-21] and transport [22-27]. Fractional integral transforms are suitable generalizations of the classical ones and were recently proposed by some researchers. For example, the fractional Fourier transforms were considered in [28, 29]. In [30], the fractional Hilbert transform was presented. The fractional Mellin transform [31,32] was proposed to be used in image encryption. The fractional wavelet transform was presented and some applications were investigated in [33-35]. In [36], the fractional Hankel transform was reported in order to research the charge-amplitude state representations.

The $Z$-transform method [1, 2, 37] was applied to handle the linear time-invariant discrete-time systems (LTI discretetime systems) and difference equations in $Z$-domain. However, the fractional derivative and integrals (the fractional PDIs) were used to transfer the fractional LTI discrete-time systems to $Z$-domain [38]. There appear signals defined on
Cantor sets, which are the most striking properties of nondifferentiable functions. The classical $Z$-transform method and PDIs did not deal with them. In order to overcome them, local fractional calculus [39-43] may be applied to handle the function defined on Cantor sets shown in Figure 1. The local fractional integral transforms via local fractional calculus theory were proposed in [44-51]. For example, local fractional Fourier transforms reported in $[40,44]$ were used to find nondifferentiable solutions for local fractional ODEs and PDEs [45-47]. Laplace transforms via local fractional calculus [40] were generalized and reported in order to solve the local fractional ODEs and PDEs [48-50].

Fractal signal processing [51-59] is a hot topic for scientists and engineers. Very recently, the concept of the $Z$-transform method via local fractional calculus was considered only in [60]. However, there is no report on signal processing by using the local fractional $Z$-transforms. The main aim of this paper is to investigate the properties of local fractional $Z$-transforms and to present some examples for processing signals defined on Cantor sets.

The paper is organized as follows. In Section 2, the concepts of local fractional complex derivatives and integrals are given. In Section 3, the notions and properties of local 


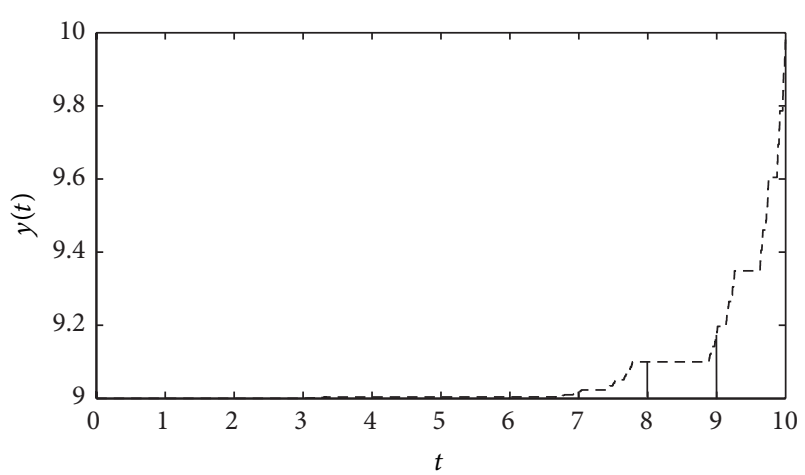

FIGURE 1: The chart of the signal $y(t)$ defined on Cantor sets.

fractional $Z$-transform method are presented. In Section 4, some examples and applications of this method are shown. Finally, Section 5 is the conclusions.

\section{Local Fractional Derivatives and Integrals of Complex Functions and Recent Results}

In this section, we introduce the concepts of local fraction derivative and integrals of complex functions. Let us first give the local fractional continuity of complex functions.

Definition 1 (see $[40,60]$ ). The function $f(z)$ is said to be local fractional continuous at $z_{0}$ if there exists

$$
\lim _{z \rightarrow z_{0}} f(z)=f\left(z_{0}\right)
$$

There is the local fractional continuous relation in the following form:

$$
f(z) \in C_{\alpha}(\Re),
$$

where

$$
\lim _{z \rightarrow z_{0}} f(z)=f\left(z_{0}\right), \quad z, z_{0} \in \Re
$$

Definition 2 (see $[40,60])$. The local fractional derivative of complex function $f(z)$ of order $\alpha$ is defined as

$$
f^{(\alpha)}(z)=\frac{d^{\alpha} f(z)}{d^{\alpha} z}=\lim _{z \rightarrow z_{0}} \frac{\Delta^{\alpha} f(z)}{\left(z-z_{0}\right)^{\alpha}}, \quad \alpha \in(0,1]
$$

where

$$
\Delta^{\alpha} f(z) \cong \Gamma(1+\alpha)\left(f(z)-f\left(z_{0}\right)\right) .
$$

If the limit of (4) exists for all $z_{0}$ in a region $\mathfrak{R}$, then the complex function $f(z)$ is said to be local fractional analytic in a region $\mathfrak{R}$.
The properties of the local fractional derivatives of some complex functions are presented as follows [40]:

$$
\begin{gathered}
\frac{d^{\alpha} z^{k \alpha}}{d z^{\alpha}}=\frac{\Gamma(1+k \alpha)}{\Gamma(1+(k-1) \alpha)} z^{(k-1) \alpha}, \\
\frac{d^{\alpha} E_{\alpha}\left(z^{\alpha}\right)}{d z^{\alpha}}=E_{\alpha}\left(z^{\alpha}\right), \\
\frac{d^{\alpha} \sin _{\alpha} z^{\alpha}}{d z^{\alpha}}=\cos _{\alpha} z^{\alpha}, \\
\frac{d^{\alpha} \cos _{\alpha} z^{\alpha}}{d z^{\alpha}}=-\sin _{\alpha} z^{\alpha},
\end{gathered}
$$

where

$$
\begin{gathered}
E_{\alpha}\left(z^{\alpha}\right)=\sum_{k=0}^{\infty} \frac{z^{\alpha k}}{\Gamma(1+k \alpha)}, \\
\sin _{\alpha} z^{\alpha}=\sum_{k=0}^{\infty} \frac{(-1)^{k} z^{\alpha(2 k+1)}}{\Gamma[1+\alpha(2 k+1)]} \\
\cos _{\alpha} z^{\alpha}=\sum_{k=0}^{\infty} \frac{(-1)^{k} z^{2 \alpha k}}{\Gamma(1+2 \alpha k)} .
\end{gathered}
$$

Definition 3 (see $[40,46-50,60]$ ). The local fractional integral of complex function $f(z)$ of order $\alpha$ along the closed contour $C$ is defined as

$$
I_{C}^{\alpha} f(z)=\frac{1}{\Gamma(1+\alpha)} \oint_{C} f(z)(d z)^{\alpha}, \quad \alpha \in(0,1] .
$$

The properties of the local fractional integrals of some complex functions are suggested as follows [40]:

$$
\begin{aligned}
& \frac{1}{\Gamma(1+\alpha)} \int_{C}(f(z)+g(z))(d z)^{\alpha} \\
& \quad=\frac{1}{\Gamma(1+\alpha)} \int_{C} f(z)(d z)^{\alpha}+\frac{1}{\Gamma(1+\alpha)} \int_{C} g(z)(d z)^{\alpha},
\end{aligned}
$$

$$
\begin{aligned}
& \frac{1}{\Gamma(1+\alpha)} \int_{C_{1}+C_{2}} f(z)(d z)^{\alpha} \\
& \quad=\frac{1}{\Gamma(1+\alpha)} \int_{C_{1}} f(z)(d z)^{\alpha}+\frac{1}{\Gamma(1+\alpha)} \int_{C_{2}} f(z)(d z)^{\alpha}, \\
& \frac{1}{\Gamma(1+\alpha)} \int_{C_{1}} f(z)(d z)^{\alpha}=-\frac{1}{\Gamma(1+\alpha)} \int_{-C_{1}} f(z)(d z)^{\alpha} .
\end{aligned}
$$

Theorem 4 (see [40]). If $f(z)$ is local fractional analytic within and on a simple closed contour $C$ and $a$ is any point interior to $C$, then we have

$$
\frac{1}{(2 \pi)^{\alpha} i^{\alpha}} \cdot\left\{\frac{1}{\Gamma(1+\alpha)} \oint_{C} \frac{f(z)}{\left(z-z_{0}\right)^{\alpha}}(d z)^{\alpha}\right\}=\frac{f\left(z_{0}\right)}{\Gamma(1+\alpha)} .
$$

Proof. See [40]. 
Definition 5 (see $[40,60]$ ). If $z_{0}$ is an isolated singular point of $f(z)$, then we have a local fractional Laurent series of $f(z)$ at $C:\left|z-z_{0}\right|<r$ given by

$$
f(z)=\sum_{i=-\infty}^{\infty} a_{k}\left(z-z_{0}\right)^{k \alpha}
$$

The coefficient $a_{-1}$ of $\left(z-z_{0}\right)^{-\alpha}$ is called the local fractional residue of $f(z)$ at $z=z_{0}$ and is frequently written as

$$
\operatorname{Re}_{z=z_{0}} s\{f(z)\}=a_{-1} .
$$

Theorem 6 (see [40]). If $f(z)$ is local fractional analytic within and on the boundary $C$ of a region $\mathfrak{R}$ except at a number of poles a within $\mathfrak{R}$, having a residue $a_{-1}$, then

$$
\frac{1}{(2 \pi)^{\alpha} i^{\alpha}} \cdot\left\{\frac{1}{\Gamma(1+\alpha)} \oint_{C} f(z)(d z)^{\alpha}\right\}=\operatorname{Re}_{z=z_{0}} s\{f(z)\} .
$$

Proof. See [40].

Theorem 7 (see [40]). If $f(z)$ is local fractional analytic within and on the boundary $C$ of a region $\mathfrak{R}$ except at a number of poles a within $\mathfrak{R}$, having numbers of residues, then

$$
\frac{1}{(2 \pi)^{\alpha} i^{\alpha}} \cdot\left\{\frac{1}{\Gamma(1+\alpha)} \oint_{C} f(z)(d z)^{\alpha}\right\}=\sum_{i=1}^{n} \operatorname{Re}_{z=z_{k}} s\{f(z)\} .
$$

Proof. See [40].

\section{Local Fractional $Z$-Transforms and Their Properties}

In this section, we give the local fractional $Z$-transforms and their properties.

Definition 8 (see [60]). Local fractional Z-transform of $f(n)$ of order $\alpha$ is defined as

$$
Z_{\alpha}\{f(n)\}=F_{\alpha}(z)=\sum_{n=\infty}^{\infty} f(n) z^{-n \alpha},
$$

where the above formula is convergent.

For a given sequence, the set $\mathfrak{R}$ of values of $z$ for which its local fractional $Z$-transform converges is called the region of convergence (ROC), namely,

$$
\sum_{n=\infty}^{\infty}\left|f(n) z^{-n \alpha}\right|<\infty
$$

The inverse formula of local fractional $Z$-transform of $f(n)$ of order $\alpha$ reads as follows (see [60]):

$$
\begin{aligned}
Z_{\alpha}^{-1}\left\{F_{\alpha}(z)\right\} & =f(n) \\
& =\frac{1}{(2 \pi i)^{\alpha} \Gamma(1+\alpha)} \oint_{C} F_{\alpha}(z) z^{(n-1) \alpha}(d z)^{\alpha},
\end{aligned}
$$

where $C$ is a counterclockwise closed fractal path encircling the origin and entirely in the region of convergence.

Let $Z_{\alpha}\{f(n)\}=F_{\alpha}(z)$ within the region of convergence $\mathfrak{R}_{1}$ and let $Z_{\alpha}\{g(n)\}=G_{\alpha}(z)$ within the region of convergence $\mathfrak{R}_{2}$.

Property 1 (linearity). We have

$$
Z_{\alpha}\{f(n)+g(n)\}=F_{\alpha}(z)+G_{\alpha}(z)
$$

within the region of convergence $\Re_{1} \cap \mathfrak{R}_{2}$.

Proof. From (15) we have

$$
\begin{aligned}
Z_{\alpha}\{f(n)+g(n)\} & =\sum_{n=\infty}^{\infty}(f(n)+g(n)) z^{-n \alpha} \\
& =Z_{\alpha}\{f(n)\}+Z_{\alpha}\{g(n)\}
\end{aligned}
$$

within the region of convergence $\mathfrak{R}_{1} \cap \mathfrak{R}_{2}$.

Property 2 (time shifting). If the variable $z$ has a useful interpretation in terms of time delay, then we have

$$
Z_{\alpha}\{f(n-k)\}=z^{-z \alpha} F_{\alpha}(z) .
$$

Proof. From (15), we have

$$
\begin{aligned}
Z_{\alpha}\{f(n-k)\} & =\sum_{n=\infty}^{\infty} f(n-k) z^{-n \alpha}=\sum_{n=\infty}^{\infty} f(n) z^{-(n+k) \alpha} \\
& =z^{-k \alpha} \sum_{n=\infty}^{\infty} f(n) z^{-n \alpha}=z^{-k \alpha} Z_{\alpha}\{f(n)\} .
\end{aligned}
$$

Property 3 (frequency modulation). We have

$$
Z_{\alpha}\left\{z_{0}^{n \alpha} f(n)\right\}=F_{\alpha}\left(\frac{z}{z_{0}}\right) .
$$

Proof. From (15), we have

$$
\begin{aligned}
Z_{\alpha}\left\{z_{0}^{n \alpha} f(n)\right\} & =\sum_{n=\infty}^{\infty} f(n) z_{0}^{n \alpha} z^{-n \alpha} \\
& =\sum_{n=\infty}^{\infty} f(n)\left(\frac{z}{z_{0}}\right)^{-n \alpha}=F_{\alpha}\left(\frac{z}{z_{0}}\right) .
\end{aligned}
$$

\section{Some Illustrative Examples}

In this section, we give some samples for nondifferentiable signals defined on Cantor sets.

Example 1. Let us consider the following signal in the form:

$$
f(n)=\delta_{\alpha}(n)= \begin{cases}1, & n=0 \\ 0, & n \neq 0\end{cases}
$$




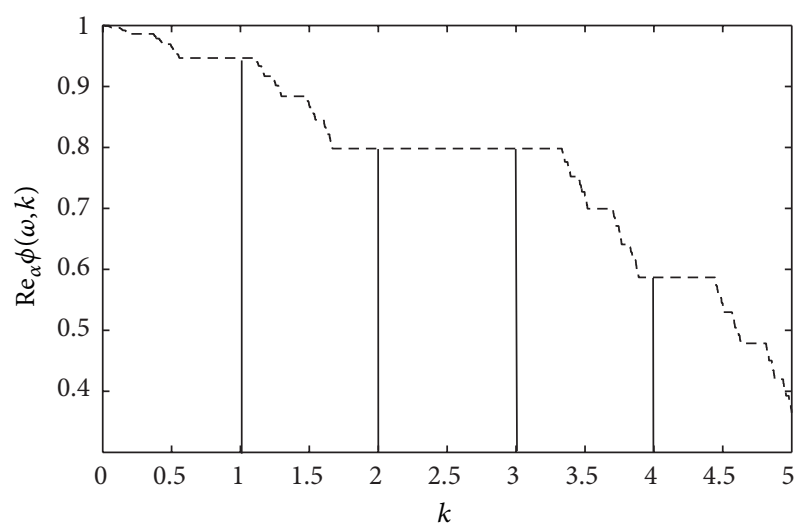

Figure 2: The graph of $\operatorname{Re}_{\alpha} \phi(\omega, k)$ with premasters $\omega=1$ and $\alpha=$ $\ln 2 / \ln 3$.

Taking local fractional $Z$-transform, we have

$$
Z_{\alpha}\{f(n)\}=Z_{\alpha}\left\{\delta_{\alpha}(n)\right\}=\sum_{n=\infty}^{\infty} \delta_{\alpha}(n) z^{-n \alpha}=1 .
$$

Example 2. We now suggest the following signal in the form:

$$
f(n)=\delta_{\alpha}(n-k)= \begin{cases}1, & n=k \\ 0, & n \neq k\end{cases}
$$

Taking local fractional $Z$-transform, we obtain

$$
Z_{\alpha}\{f(n)\}=Z_{\alpha}\left\{\delta_{\alpha}(n-k)\right\}=\sum_{n=\infty}^{\infty} \delta_{\alpha}(n-k) z^{-n \alpha}=z^{-k \alpha}
$$

When $z^{\alpha}=E_{\alpha}\left(j^{\alpha} \omega^{\alpha}\right)$ with the imaginary unit $j^{\alpha}[40,44-50]$, we get

$$
\begin{aligned}
\phi(\omega, k) & =Z_{\alpha}\{f(n)\}=E_{\alpha}\left(-j^{\alpha} \omega^{\alpha} k^{\alpha}\right) \\
& =\cos _{\alpha}\left(\omega^{\alpha} k^{\alpha}\right)-j^{\alpha} \sin _{\alpha}\left(\omega^{\alpha} k^{\alpha}\right) .
\end{aligned}
$$

Hence, from (28), we get

$$
\begin{aligned}
& \operatorname{Re}_{\alpha} \phi(\omega, k)=\cos _{\alpha}\left(\omega^{\alpha} k^{\alpha}\right), \\
& \operatorname{Im}_{\alpha} \phi(\omega, k)=-\sin _{\alpha}\left(\omega^{\alpha} k^{\alpha}\right)
\end{aligned}
$$

with the real part graph in Figure 2 and imaginary part graph in Figure 3.

Example 3. There is the signal in the following form:

$$
f(n)=\delta_{\alpha}(n-k)+\delta_{\alpha}(n+k)= \begin{cases}1, & n= \pm k \\ 0, & n \neq \pm k .\end{cases}
$$

Taking local fractional $Z$-transform, we have

$$
\begin{aligned}
Z_{\alpha}\{f(n)\} & =Z_{\alpha}\left\{\delta_{\alpha}(n-k)+\delta_{\alpha}(n+k)\right\} \\
& =\sum_{n=\infty}^{\infty}\left(\delta_{\alpha}(n-k)+\delta_{\alpha}(n+k)\right) z^{-n \alpha}=z^{-k \alpha}+z^{k \alpha} .
\end{aligned}
$$

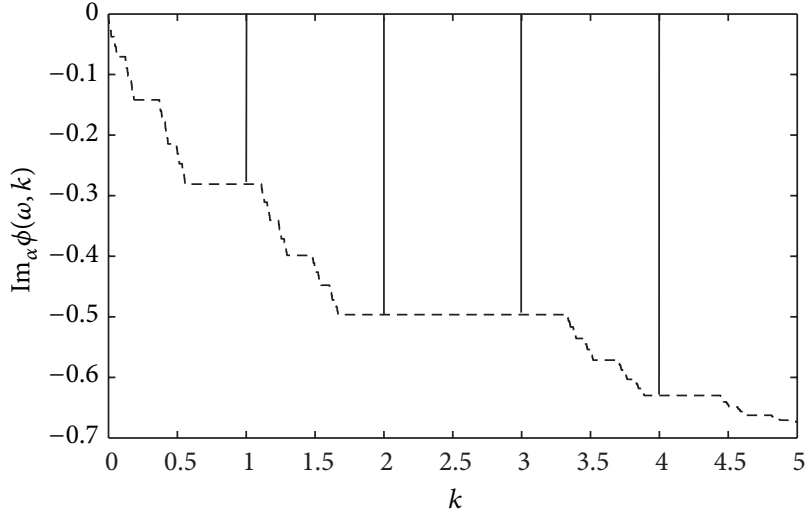

Figure 3: The plot of $\operatorname{Im}_{\alpha} \phi(\omega, k)$ with premasters $\omega=1$ and $\alpha=$ $\ln 2 / \ln 3$.

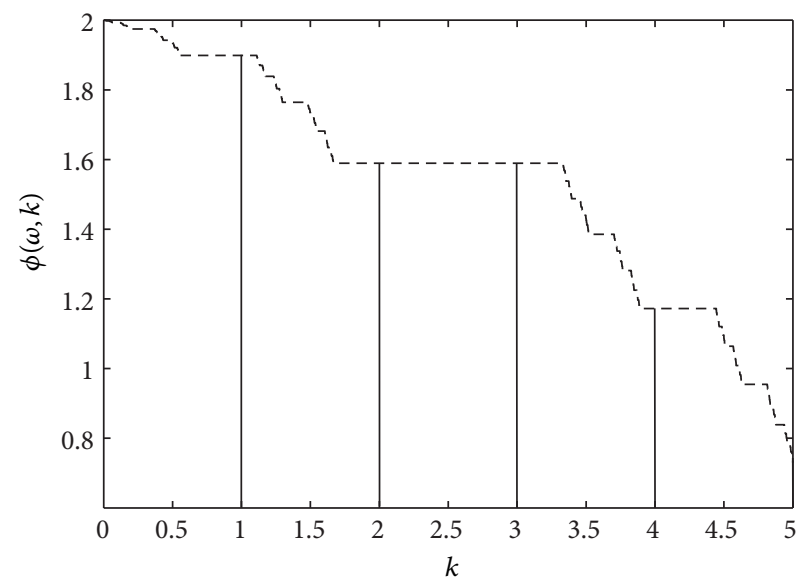

FIgURE 4: The map of $\phi(\omega, k)$ with premasters $\omega=1$ and $\alpha=$ $\ln 2 / \ln 3$.

When $z^{\alpha}=E_{\alpha}\left(j^{\alpha} \omega^{\alpha}\right)$, we get

$$
\phi(\omega, k)=Z_{\alpha}\{f(n)\}=E_{\alpha}\left(j^{\alpha} \omega^{\alpha} k^{\alpha}\right)=2 \cos _{\alpha}\left(\omega^{\alpha} k^{\alpha}\right)
$$

with the graph of $\phi(\omega, k)$ shown in Figure 4.

Example 4. We have the following signal in the form:

$$
f(n)= \begin{cases}a^{n \alpha}, & a \geq 0 \\ 0, & a<0\end{cases}
$$

Local fractional $Z$-transform gives the following form:

$$
Z_{\alpha}\{f(n)\}=Z_{\alpha}\left\{a^{n \alpha}\right\}=\sum_{n=0}^{\infty} a^{n \alpha} z^{-n \alpha}=\sum_{n=0}^{\infty}\left(\frac{z}{a}\right)^{-n \alpha}
$$

with the region of convergence $|z|>|a|$.

Example 5. We consider the following signal in the form:

$$
f(n)= \begin{cases}0, & a \geq 0 \\ a^{n \alpha}, & a<0\end{cases}
$$


Taking local fractional $Z$-transform, we arrive at the following form:

$$
Z_{\alpha}\{f(n)\}=Z_{\alpha}\left\{a^{n \alpha}\right\}=\sum_{n=\infty}^{0} a^{n \alpha} z^{-n \alpha}=\sum_{n=\infty}^{0}\left(\frac{z}{a}\right)^{-n \alpha}
$$

with the region of convergence $|z|<|a|$.

Example 6. We present the following signal in the form:

$$
f(n)= \begin{cases}b^{n \alpha}, & a \geq 0 \\ a^{n \alpha}, & a<0\end{cases}
$$

Local fractional $Z$-transform gives the following form:

$$
\begin{aligned}
Z_{\alpha}\{f(n)\} & =Z_{\alpha}\left\{a^{n \alpha}+a^{n \alpha}\right\}=\sum_{n=0}^{\infty} b^{n \alpha} z^{-n \alpha}+\sum_{n=\infty}^{0} a^{n \alpha} z^{-n \alpha} \\
& =\sum_{n=0}^{\infty}\left(\frac{z}{b}\right)^{-n \alpha}+\sum_{n=\infty}^{0}\left(\frac{z}{a}\right)^{-n \alpha}
\end{aligned}
$$

with the region of convergence $|b|<|z|<|a|$.

\section{Conclusions}

In this work, we investigated the local fractional $Z$-transforms based on the local fractional complex calculus and some properties are also obtained. Some illustrative examples were also given. The obtained results show the accuracy and efficiency of the presented method.

\section{Conflict of Interests}

The authors declare that they have no competing interests in this paper.

\section{Acknowledgment}

This work was supported by Science and Technology Commission Planning Project of Jiangsu Province (no. BE2013737).

\section{References}

[1] B. Davies, Integral Transforms and Their Applications, Texts in Applied Mathematics, Springer, New York, NY, USA, 2002.

[2] L. Debnath and D. Bhatta, Integral Transforms and Their Applications, CRC Press, New York, NY, USA, 2010.

[3] V. E. Tarasov, Fractional Dynamics, Nonlinear Physical Science, Springer, New York, NY, USA, 2010.

[4] R. Herrmann, Fractional Calculus: An Introduction for Physicists, World Scientific, Singapore, 2011.

[5] A. B. Malinowska and D. F. M. Torres, Introduction to the Fractional Calculus of Variations, Imperial College Press, London, UK, 2012.
[6] M. D. Ortigueira, Fractional Calculus for Scientists and Engineers, Lecture Notes in Electrical Engineering, Springer, New York, NY, USA, 2011.

[7] F. Mainardi, Fractional Calculus and Waves in Linear Viscoelasticity, Imperial College Press, London, UK, 2010.

[8] J. Sabatier, O. P. Agrawal, and J. A. T. Machado, Advances in Fractional Calculus: Theoretical Developments and Applications in Physics and Engineering, Springer, New York, NY, USA, 2007.

[9] A. A. Kilbas, H. M. Srivastava, and J. J. Trujillo, Theory and Applications of Fractional Differential Equations, vol. 204 of North-Holland Mathematics Studies, Elsevier Science B.V., Amsterdam, The Netherlands, 2006.

[10] I. Podlubny, Fractional Differential Equations, vol. 198 of Mathematics in Science and Engineering, Academic Press, San Diego, Calif, USA, 1999.

[11] B. J. West, M. Bologna, and P. Grigolini, Physics of Fractal Operators, Institute for Nonlinear Science, Springer, New York, NY, USA, 2003.

[12] R. Metzler and J. Klafter, “The random walk's guide to anomalous diffusion: a fractional dynamics approach," Physics Reports, vol. 339, no. 1, pp. 1-77, 2000.

[13] L. Miller, "On the controllability of anomalous diffusions generated by the fractional Laplacian," Mathematics of Control, Signals, and Systems, vol. 18, no. 3, pp. 260-271, 2006.

[14] B. J. West, P. Grigolini, R. Metzler, and T. F. Nonnenmacher, "Fractional diffusion and Lévy stable processes", Physical Review E, vol. 55, no. 1, pp. 99-106, 1997.

[15] V. V. Uchaikin, "Anomalous diffusion and fractional stable distributions," Journal of Experimental and Theoretical Physics, vol. 97, no. 4, pp. 810-825, 2003.

[16] Y. Zhang, D. A. Benson, M. M. Meerschaert, E. M. LaBolle, and H. P. Scheffler, "Random walk approximation of fractionalorder multiscaling anomalous diffusion," Physical Review E, vol. 74, no. 2, Article ID 026706, 2006.

[17] Q. Yang, I. Turner, F. Liu, and M. Ilic, "Novel numerical methods for solving the time-space fractional diffusion equation in two dimensions," SIAM Journal on Scientific Computing, vol. 33, no. 3, pp. 1159-1180, 2011.

[18] R. L. Magin, O. Abdullah, D. Baleanu, and X. J. Zhou, "Anomalous diffusion expressed through fractional order differential operators in the Bloch-Torrey equation," Journal of Magnetic Resonance, vol. 190, no. 2, pp. 255-270, 2008.

[19] H. Sun, W. Chen, C. Li, and Y. Chen, "Fractional differential models for anomalous diffusion," Physica A, vol. 389, no. 14, pp. 2719-2724, 2010.

[20] M. Xu and W. Tan, "Theoretical analysis of the velocity field, stress field and vortex sheet of generalized second order fluid with fractional anomalous diffusion," Science in China A, vol. 44, no. 11, pp. 1387-1399, 2001.

[21] A. V. Chechkin, R. Gorenflo, and I. M. Sokolov, "Fractional diffusion in inhomogeneous media," Journal of Physics A, vol. 38, no. 42, pp. L679-L684, 2005.

[22] G. M. Zaslavsky, "Chaos, fractional kinetics, and anomalous transport," Physics Reports, vol. 371, no. 6, pp. 461-580, 2002.

[23] B. Berkowitz, J. Klafter, R. Metzler, and H. Scher, "Physical pictures of transport in heterogeneous media: advectiondispersion, random-walk, and fractional derivative formulations," Water Resources Research, vol. 38, no. 10, pp. 9-1-9-12, 2002.

[24] J. D. Seymour, J. P. Gage, S. L. Codd, and R. Gerlach, "Anomalous fluid transport in porous media induced by biofilm 
growth," Physical Review Letters, vol. 93, no. 19, Article ID 198103, 4 pages, 2004.

[25] Y. Luchko and A. Punzi, "Modeling anomalous heat transport in geothermal reservoirs via fractional diffusion equations," GEM-International Journal on Geomathematics, vol. 1, no. 2, pp. 257-276, 2011.

[26] J. Klafter, S. C. Lim, and R. Metzler, Fractional Dynamics, Recent Advances, World Scientific, Singapore, 2012.

[27] P. Chun and J. Inoue, "Yet another possible mechanism for anomalous transport: theory, numerical method, and experiments," KSCE Journal of Civil Engineering, vol. 16, no. 1, pp. 4553, 2012.

[28] A. C. McBride and F. H. Kerr, "On Namias's fractional Fourier transforms," IMA Journal of Applied Mathematics, vol. 39, no. 2, pp. 159-175, 1987.

[29] H. M. Ozaktas and O. Aytür, "Fractional Fourier domains," Signal Processing, vol. 46, no. 1, pp. 119-124, 1995.

[30] A. W. Lohmann, D. Mendlovic, and Z. Zalevsky, "Fractional Hilbert transform," Optics Letters, vol. 21, no. 4, pp. 281-283, 1996.

[31] N. Zhou, Y. Wang, and L. Gong, "Novel optical image encryption scheme based on fractional Mellin transform," Optics Communications, vol. 284, no. 13, pp. 3234-3242, 2011.

[32] Y. Luchko and V. Kiryakova, "The Mellin integral transform in fractional calculus," Fractional Calculus and Applied Analysis, vol. 16, no. 2, pp. 405-430, 2013.

[33] D. Mendlovic, Z. Zalevsky, D. Mas, J. García, and C. Ferreira, "Fractional wavelet transform," Applied Optics, vol. 36, no. 20, pp. 4801-4806, 1997.

[34] J. Shi, N. Zhang, and X. Liu, "A novel fractional wavelet transform and its applications," Science China: Information Sciences, vol. 55, no. 6, pp. 1270-1279, 2012.

[35] E. Dinç and D. Baleanu, "Fractional wavelet transform for the quantitative spectral resolution of the composite signals of the active compounds in a two-component mixture," Computers \& Mathematics with Applications, vol. 59, no. 5, pp. 1701-1708, 2010.

[36] H. Y. Fan, "Fractional Hankel transform studied by chargeamplitude state representations and complex fractional Fourier transformation," Optics Letters, vol. 28, no. 22, pp. 2177-2179, 2003.

[37] E. I. Jury, Theory and Applications of the Z-Transform Method, John Wiley \& Sons, New York, NY, USA, 1964.

[38] J. A. T. Machado, "Analysis and design of fractional-order digital control systems," Systems Analysis Modelling Simulation, vol. 27, no. 2-3, pp. 107-122, 1997.

[39] X. J. Yang, Advanced Local Fractional Calculus and Its Applications, World Science, New York, NY, USA, 2012.

[40] X. J. Yang, Local Fractional Functional Analysis and Its Applications, Asian Academic Publisher, Hong Kong, China, 2011.

[41] X. J. Yang and D. Baleanu, "Fractal heat conduction problem solved by local fractional variation iteration method," Thermal Science, vol. 17, no. 2, pp. 625-628, 2013.

[42] X. J. Yang, D. Baleanu, and W. P. Zhong, "Approximation solutions for diffusion equation on Cantor time-space," Proceeding of the Romanian Academy A, vol. 14, no. 2, pp. 127-133, 2013.

[43] Y. Zhao, D. Baleanu, C. Cattani, D. F. Cheng, and X. J. Yang, "Maxwell's equations on Cantor sets: a local fractional approach," Advances in High Energy Physics, vol. 2013, Article ID 686371, 6 pages, 2013.
[44] X. J. Yang, D. Baleanu, and J. A. T. Machado, "Mathematical aspects of the Heisenberg uncertainty principle within local fractional Fourier analysis," Boundary Value Problems, no. 1, article 131, 20132013.

[45] F. Gao, W. P. Zhong, and X. M. Shen, "Applications of YangFourier transform to local fractional equations with local fractional derivative and local fractional integral," Advanced Materials Research, vol. 461, pp. 306-310, 2012.

[46] Y. Zhao, D. Baleanu, M. C. Baleanu, D. F. Cheng, and X. J. Yang, "Mappings for special functions on Cantor sets and special integral transforms via local fractional operators," Abstract and Applied Analysis, vol. 2013, Article ID 316978, 6 pages, 2013.

[47] J.-H. He, "Asymptotic methods for solitary solutions and compactons," Abstract and Applied Analysis, vol. 2012, Article ID 916793, 130 pages, 2012.

[48] C. F. Liu, S. S. Kong, and S. J. Yuan, "Reconstructive schemes for variational iteration method within Yang-Laplace transform with application to fractal heat conduction problem," Thermal Science, vol. 17, no. 3, pp. 715-721, 2013.

[49] C. G. Zhao, A. M. Yang, H. Jafari, and A. Haghbin, "The YangLaplace transform for solving the IVPs with local fractional derivative," Abstract and Applied Analysis, vol. 2014, Article ID 386459, 5 pages, 2014.

[50] S. Q. Wang, Y. J. Yang, and H. K. Jassim, "Local fractional function decomposition method for solving inhomogeneous wave equations with local fractional derivative," Abstract and Applied Analysis, vol. 2014, Article ID 176395, 7 pages, 2014.

[51] M. Li and W. Zhao, "On bandlimitedness and lag-limitedness of fractional Gaussian noise," Physica A, vol. 392, no. 9, pp. 19551961, 2013.

[52] C. Cattani, "Harmonic wavelet approximation of random, fractal and high frequency signals," Telecommunication Systems, vol. 43, no. 3-4, pp. 207-217, 2010.

[53] J. Nagler and J. C. Claussen, " $1 / f^{\alpha}$ spectra in elementary cellular automata and fractal signals," Physical Review E, vol. 71, no. 6, Article ID 067103, 4 pages, 2005.

[54] A. Gupta and S. Joshi, "Variable step-size LMS algorithm for fractal signals," IEEE Transactions on Signal Processing, vol. 56, no. 4, pp. 1411-1420, 2008.

[55] B. J. West, Fractal Physiology and Chaos in Medicine, World Scientific, Singapore, 2013.

[56] M. Li, "Power spectrum of generalized fractional Gaussian noise," Advances in Mathematical Physics, vol. 2013, Article ID 315979, 3 pages, 2013.

[57] M. Li, "Modeling autocorrelation functions of long-range dependent teletraffic series based on optimal approximation in Hilbert space - a further study," Applied Mathematical Modelling, vol. 31, no. 3, pp. 625-631, 2007.

[58] M. Li and S. C. Lim, "Power spectrum of generalized Cauchy process," Telecommunication Systems, vol. 43, no. 3-4, pp. 219222, 2010.

[59] M. Li, "A class of negatively fractal dimensional Gaussian random functions," Mathematical Problems in Engineering, vol. 2011, Article ID 291028, 18 pages, 2011.

[60] G. Yi, "Local fractional Z transform in fractal space," Advances in Digital Multimedia, vol. 1, no. 2, pp. 96-102, 2012. 


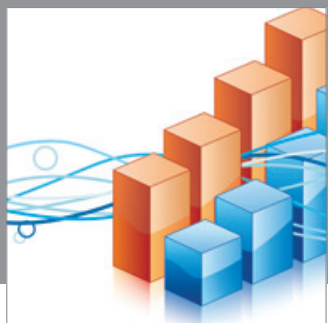

Advances in

Operations Research

mansans

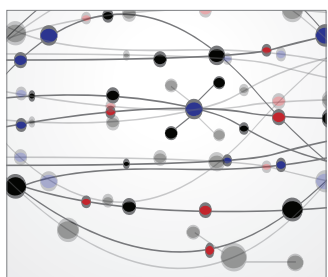

The Scientific World Journal
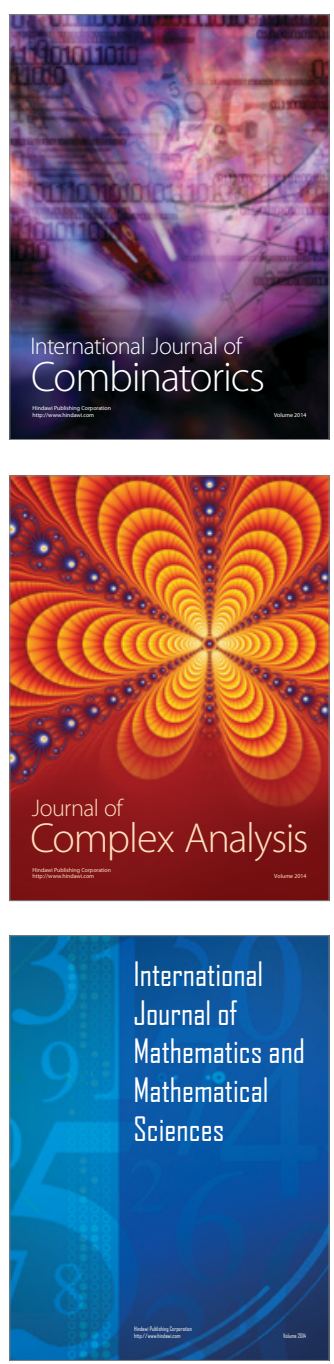
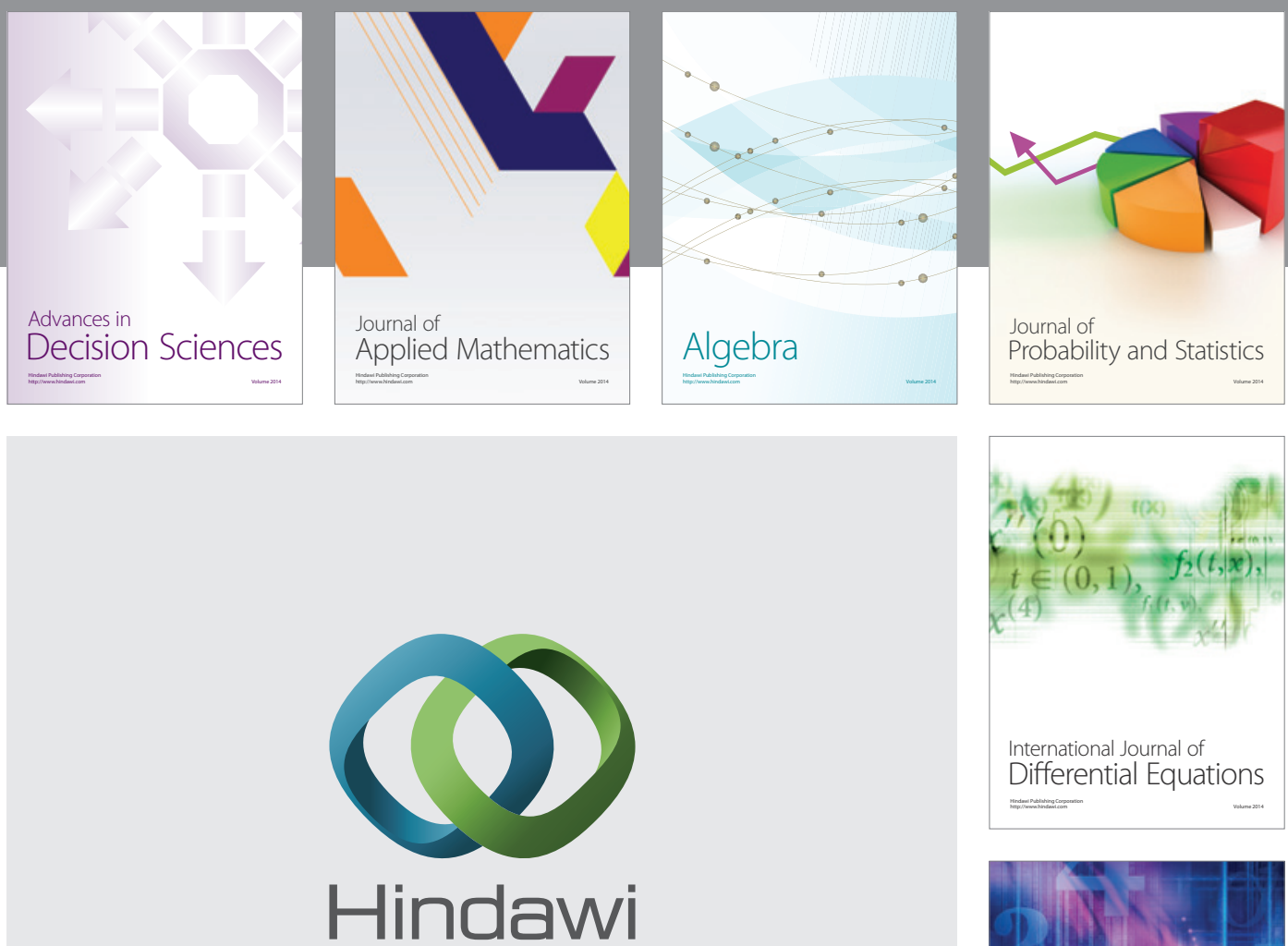

Submit your manuscripts at http://www.hindawi.com
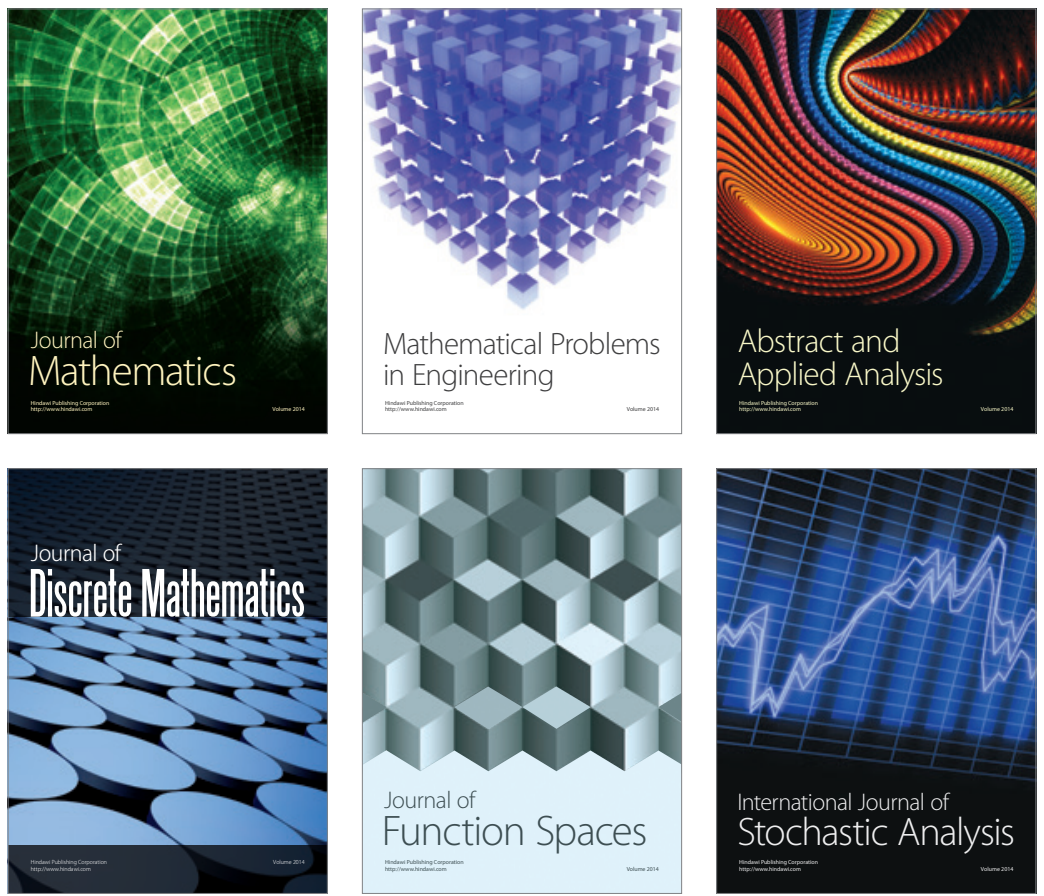

Journal of

Function Spaces

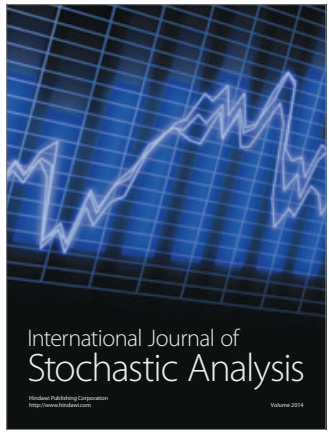

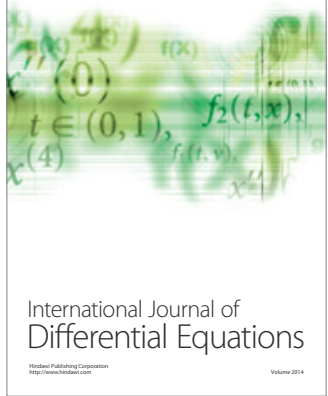
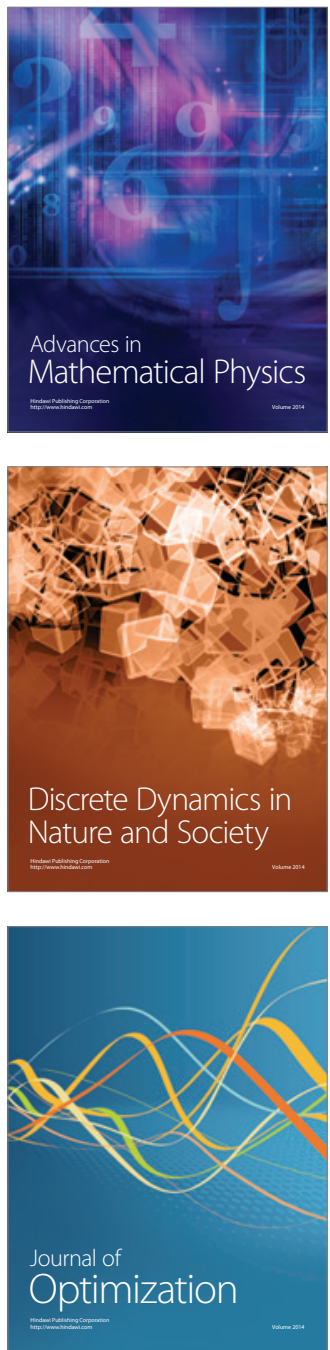